\title{
The Hellenic Open University Cosmic Ray Telescope: Research and Educational Activities
}

\author{
A. Leisos ${ }^{1, a}$, T. Avgitas ${ }^{1,2}$, G. Bourlis ${ }^{1}$, G.K. Fanourakis ${ }^{3}$, I. Gkialas ${ }^{4}$, I. Manthos M.5 $^{4,}$ A. \\ Stamelakis $^{1,6}$, A.G.Tsirigotis ${ }^{1}$, and S.E. Tzamarias ${ }^{1,5}$ \\ ${ }^{1}$ Physics Laboratory, School of Science and Technology, Hellenic Open University, Patras, Greece \\ ${ }^{2}$ Laboratory APC, University Paris Diderot - Paris VII, Paris, France \\ ${ }^{3}$ Institute of Nuclear and Particle Physics, NCSR Demokritos, Athens, Greece \\ ${ }^{4}$ Department of Financial and Management Engineering, University of the Aegean, Chios, Greece \\ ${ }^{5}$ Department of Physics, Aristotle University of Thessaloniki, Thessaloniki, Greece \\ ${ }^{6}$ Department of Physics, University of Patras, Patras, Greece
}

\begin{abstract}
The Hellenic Open University Cosmic Ray Telescope consists of three autonomous stations installed at the University Campus in the city of Patras. Each station comprises three large $\left(\approx 1 \mathrm{~m}^{2}\right)$ plastic scintillators and one or more Codalema type $\mathrm{RF}$ antennas detecting Extensive Air Showers (EAS), originating from primary particles with energy greater than $10 \mathrm{TeV}$. The operation and the performance of the Telescope is presented briefly, emphasising the educational activities foreseen in the framework of the HEllenic LYceum Cosmic Observatories Network (HELYCON).
\end{abstract}

\section{Introduction}

Cosmic rays were the first source of sub-atomic particles and played a major role in the early exploration of elementary particles. Nowadays, a century after their discovery, cosmic rays are still of great scientific interest for many reasons: the wide energy spectrum, the origin of the high energy cosmic rays, the acceleration mechanisms, the composition of the primary particles, etc. When a primary particle enters the earth's atmosphere, it collides with a nucleus and the products of the interaction produce new particles in secondary interactions and so on. As a result, a shower of particles is formed travelling with the speed of light in the atmosphere. As the shower develops, the number of secondaries increases reaching a maximum and then the shower dies out. For initial energies higher than $\approx 10^{13} \mathrm{eV}$ the shower maximum is deep inside the atmosphere and therefore many particles reach the ground, spreading up to several thousands of square meters for very energetic showers. There are many detection techniques for these extended air showers: fluorescence detectors which measure the excitation of air molecules, cherenkov telescopes that measure the electromagnetic component of the shower, radio antennas which measure the radio signal produced by energetic primaries as well as scintillator counters and tracking detectors that detect particles at the ground level or even below. The most simple and common technique is the use of ground based sparse arrays of scintillators. The availability of such scintillator counters decommissioned by large particle physics experiments, the

ae-mail: leisos@eap.gr 
public interest on cosmic rays and the initiatives of physics departments to spread the scientific knowledge and achievements to the public, resulted in the birth of educational cosmic ray telescopes mainly in the United States and Canada. The idea is that scintillator counters positioned on the roofs of high schools can form a large sparse array of detectors across big cities, covering an area of many square kilometers. After the initial project in Alberta of Canada in 1993, many more projects started in the United States in the beginning of the $21^{\text {th }}$ century and now a lot of educational cosmic ray telescopes across America and Europe exist to serve research and education in this field.

\section{Educational Cosmic Ray Telescopes}

A typical educational array site consists of three or four plastic scintillator detectors, connected to photomultiplier tubes (PMT) and read out by custom built electronics. The detectors are placed a few meters apart on the roof of a school, college or university building. By requiring three or more of these detectors to register particles within a narrow time window, one can distinguish between the relatively high flux of individual particles from low energy cosmics and the lower rate of extensive air showers caused by higher energy primaries. Measuring the relative times that the shower front passes through the particle detectors and using triangulation, the direction of the shower axis and consequently of the primary particle can be reconstructed with an accuracy of a few degrees. Using the global positioning system (GPS) to provide an absolute time reference, data from distant array sites can be combined.

Such an educational array allows for many student and teacher activities like:

1. Classroom lessons for the history of cosmic rays, particle interaction with matter, detection techniques etc.

2. The construction of the array (i.e. polishing and cleaning the scintillator, gluing the PMTs, wrapping the scintillator, etc).

3. Calibration and testing of the PMTs and the scintillator counters.

4. Operating and Monitoring the array.

5. Shower reconstruction and Data Analysis (local or combined data from several stations).

6. Workshops for the presentation of the results, public lectures for outreach etc.

There are many educational cosmic ray telescopes across America and Europe [1]. In Greece the relevant pilot project started at the Hellenic Open University (HOU) and it is called HELYCON (Hellenic Lyceum Cosmic Observatories Network) [2]. The project evolved in parallel with the design of the KM3NeT underwater neutrino telescope where a scintillator counter array was proposed as part of the calibration system [3,4]. Using existing laboratory infrastructure and small university fundings, 12 scintillator counters (HELYCON Detector Modules) were designed, constructed, tested and operated at the Physics Laboratory of HOU. The detectors were also installed and operated during summer schools in the area of Western Greece.

\section{The Hellenic Open University Cosmic Ray Telescope}

The Hellenic Open University Cosmic Ray Telescope was constructed by the ASTRONEU Collaboration in the framework of a four year national research program. It consists of 9 HELYCON Detector Modules (HDM) and 6 Radio Frequency antennas (RFA), arranged in three autonomous stations. The stations were installed in 2014 and are still in operation at the University Campus in Patras in Western 


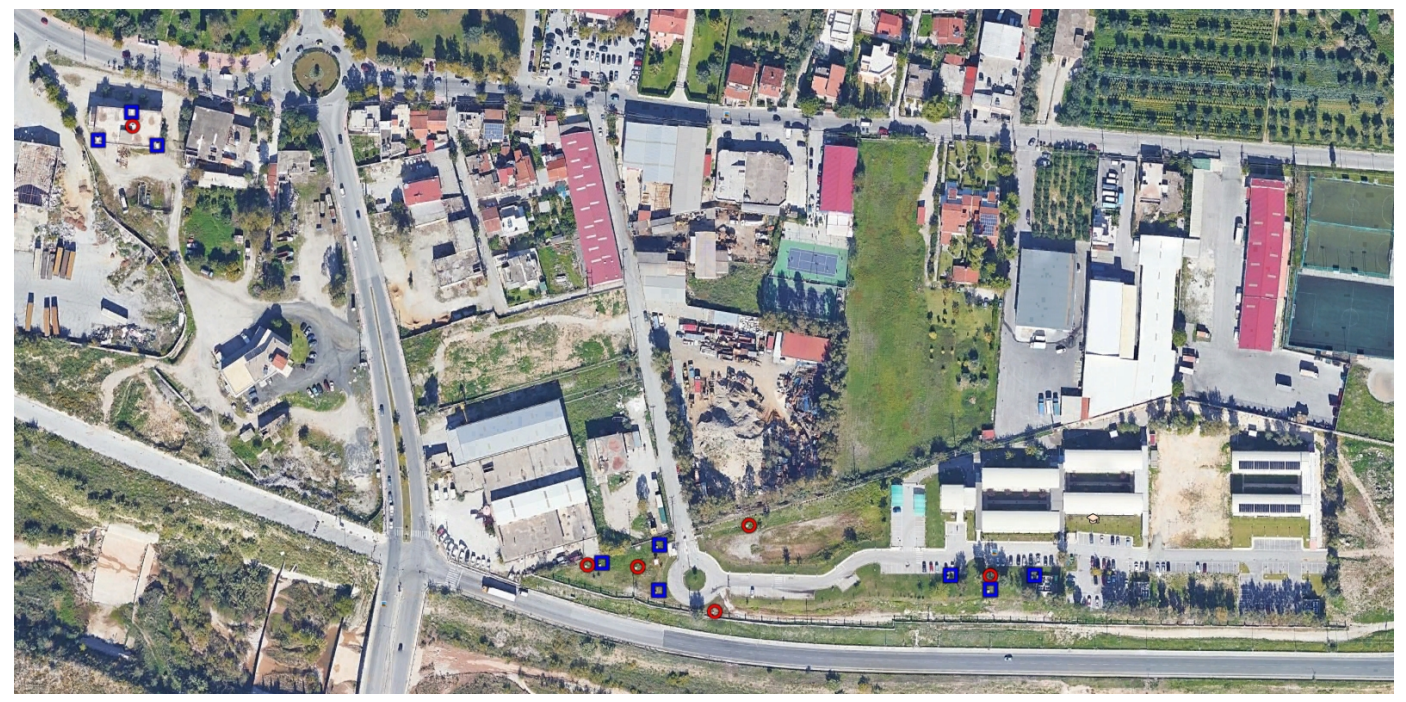

Figure 1. A satellite view of the Hellenic Open University Cosmic Ray Telescope. The blue and red coloured indicators show the positions of the scintillator counters and the RF antennas respectively.

Greece (figure 1). All stations consists of three HDM and one RFA, whilst since 2016 three more RFA were added to one of the stations. Each HDM [2] is built of 160 scintillating tiles covering an area of about $1 \mathrm{~m}^{2}$. The light produced by the scintillator is guided to a single PMT using 96 embedded wavelength shifting fibers (WLS). Power is supplied by a dc-dc converter positioned near the PMT inside the detector which is controlled and monitored using an external USB I/O device. The scintillator tiles are wrapped with reflective paper and the whole detector with aluminum foils and then it is placed inside a waterproof and lightproof box.

The signals of the three HDM PMTs are acquired by a Quarknet DAQ board [5], which employs a 3-fold majority trigger logic, while a trigger out signal is sent to the RFA of the station. The Quarknet DAQ board measures the times of the crossings of the PMT waveforms with adjustable predefined thresholds. The time of the first crossing defines the timing of the pulse, while the time that the pulse remains above the threshold (Time over Threshold - ToT) is used to estimate the pulse size. The collected data are timestamped using a GPS module and transferred to a PC, which also hosts the control and monitoring software (figure 2). The Quarknet DAQ board is operated through the USB port of the $\mathrm{PC}$ and it has 4 input channels with amplification. The times of the threshold crossings are digitised with an accuracy of $1.25 \mathrm{~ns}$. It was designed at Fermilab and it is used by the majority of the educational arrays in USA and Europe. The ASTRONEU Collaboration has designed a new low cost DAQ board with better resolution and more adjustable thresholds to replace the Quaknet DAQ board. The prototype [6] includes two input channels with three adjustable thresholds for channel A and one for channel B. It is based on an ultra-high performance time to digital converter for the time tagging of the crossings of the pulses with the adjustable thresholds with an accuracy of $0.1 \mathrm{~ns}$. The board is USB connected to the hosting computer and an external GPS receiver is employed for the absolute time reference.

The bipolar RF antenna was designed and constructed by the CODALEMA [7] experiment and is capable of detecting the radio signals produced by high energy showers (originating by primaries with energy exceeding $10^{17} \mathrm{eV}$ ). In the HOU cosmic ray telescope the antennas are operated in external- 
trigger mode using the trigger-out signal of the corresponding station Quarknet board. The RFA digitisation electronics sample the waveform at $1 \mathrm{GHz}$ and 2560 samples are recorded. The signals are processed in order to keep only frequencies in the range 20-80 MHz. Further signal processing procedures that check the EW vs NS polarisation, the pulse rise time and the cumulative signal are applied to characterise the event as cosmic or not [8].
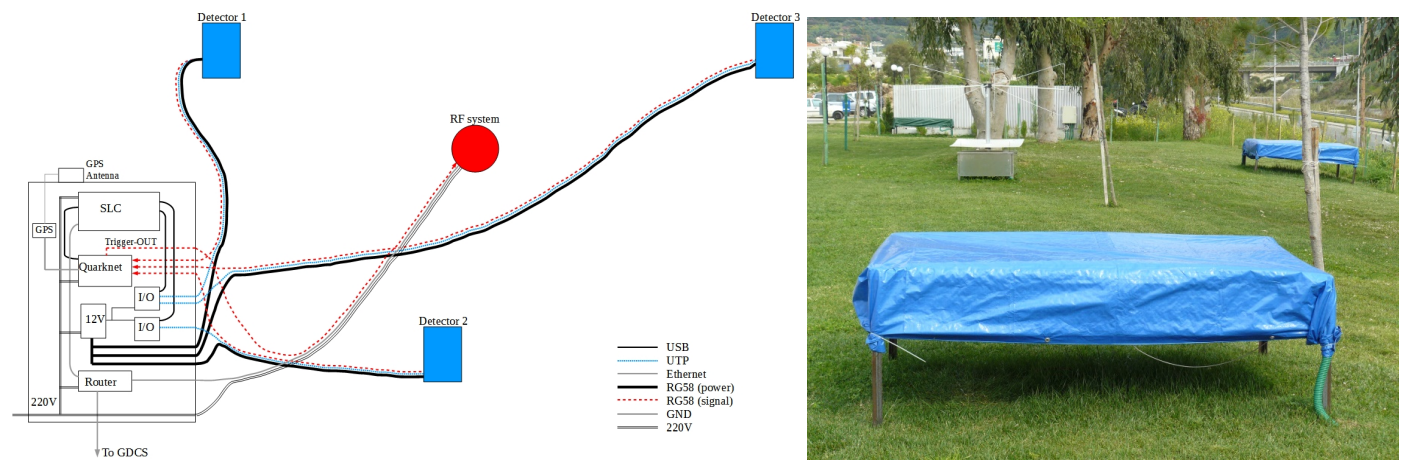

Figure 2. On the left the schematic of a station is shown. Signals from the three scintillators are acquired by the Quarknet board and a 3-fold coincidence is applied. A trigger out signal is sent to the RF-antenna electronics, which has its own control and DAQ system. GPS timestamp is provided by both the Quarknet board and the antenna hardware. Data are stored locally to a laptop and once a day they are transferred to the central server via Ethernet. The photo on the right shows one of the stations at the HOU campus. The three blue boxes enclose the scintillator counters and the RF antenna is positioned among them.

Before deployment several calibration procedures [9] and tests are performed on the individual components and on the station as a whole. The PMTs are calibrated for gain, single photoelectron (pe) characteristics and dark count rate. The scintillator tests include the determination of the Minimum Ionising Particle (MIP) light yield, the relative strength and the relative timing of the signal with respect to the incident position on the counter (uniformity), as well as the effect of the WLS fibers (absorption and emission process). Then the functional characteristics of the Quarknet DAQ board (threshold values, timing and ToT accuracy, trigger logic) and the cabling effects are determined. Finally, the last test includes the operation of two stations side by side. Angular distributions, charge collection efficiencies, waveform shapes etc are compared and if needed the relevant parameters are tuned in the MC simulation.

Since the only available information from the DAQ system is the times of the crossings of the waveform with the threshold, we utilise the ToT technique [10] in order to estimate the collected charge and the pulse height of the waveforms with good accuracy ${ }^{1}$. Parametrisation curves of the charge and the pulse height as a function of the ToT value are produced for several different voltage threshold values [9]. These parameterised quantities are used for slewing correction and the estimation of the corresponding resolution, but they are also very helpful for the definition of quality criteria and for the interpretation of the results.

The offline software used is part of the Hellenic Open University Simulation and Reconstruction software (HOURS) [11, 12] with primary use in neutrino telescopy. The simulation software describes in detail the detector components and is interfaced with the CORSIKA package [13], which simulates

\footnotetext{
${ }^{1}$ The accuracy depends on the size of the pulse and for small pulses is around $20 \%$, while for larger pulses is better than $15 \%$.
} 
the development of the EAS and provides the particle information at the detector level. The HOURS software uses parameterisations for the scintillation process and the effect of the WLS fibers which are determined either by detailed simulation using the GEANT4 package [14] or by using the results of the calibration procedure. The photons reaching the PMT are used to generate the photoelectrons and the PMT response pulse. Finally, the signal transmission (cable effects) and the digitisation electronics functionality are simulated to produce the raw data. The reconstruction and monitoring software applies the signal processing procedures, the quality criteria and finally the shower angular parameters are estimated and performance plots are produced.

We have analysed over one and a half year of data from all the stations. The analysis of the data and the performance of the telescope is summarised in table 1. For each station the rate of the selected shower events (events passing the quality criteria), the distribution of the ToT value and the estimated number of MIPs registered by each HDM, as well as the angular distributions (zenith and azimuth angle) of the reconstructed showers, were found to be in excellent agreement with the MC expectations[15]. The shower angular direction (azimuth and zenith angle) were reconstructed using the plane particle front approximation ${ }^{2}$ using triangulation. Shower events that trigger the closest pair of stations were also analysed. These showers have energy greater than $10^{15} \mathrm{eV}$ and the majority of the impact points is distributed between the two stations (on average $100 \mathrm{~m}$ from each station centre). In these high energy showers the particles are spread further from the shower axis and consequently the shower front curvature must be taken into account expecting extra delays depending on the position of the detectors relative to the shower axis. The extra delay is estimated from MC samples using a classification parameter, which is used to estimate the position of the impact point of the shower i.e. if the impact point is closer to one station than to the other. By applying this correction the median of the 3-D angle ${ }^{3}$ resolution was improved by one degree compared to the standard plane particle front approximation.

The shower events triggering both stations have a substantial fraction of high energy showers that give radio signals, which can be captured by the RF antennas. Although such antennas are supposed to be operated in a radio-quite environment, the trigger signal of the HDMs and the application of quality criteria proved to be sufficient in order to select RF signals originating from cosmic events[8]. These events were collected with an older configuration of the telescope where only one RFA participated in each station. However, the timing of the two RF signals were found to be consistent with the expected values estimated from the reconstructed direction of the showers using the HDM only and the positions of the RF antennas. In the new configuration where 3 more RFA were installed in one of the stations will enable the reconstruction of the shower direction using only the RF signals.

\section{Educational activities with the Hellenic Open University Cosmic Ray Telescope}

Even though research activities are still on-going with the HOU cosmic ray telescope, a wide outreach program is foreseen to get high school teachers and students involved. During the last months we have given many lectures and seminars for the HOU cosmic ray telescope, we have participated in many workshops and summer schools of the Greek Association of Physicists followed by "hands on" activities, but we have also contributed to high publicity events, where students had the opportunity to visit the HOU Telescope and the Physics Laboratory.

\footnotetext{
${ }^{2}$ Due to the small separation of the HDM $(\approx 26 \mathrm{~m})$ in each station, the energy threshold of the reconstructed showers is very low $(\approx 20 \mathrm{TeV})$ and the impact points of the showers are close (on average $30 \mathrm{~m}$ ) to the centre of the station. In these showers the deviation of the particle shower front from the plane particle front approximation is very small compared to the shower front thickness.

${ }^{3}$ The angle between the reconstructed and the true direction of the shower.
} 
Table 1. Performance parameters of the Hellenic Open University Cosmic Ray Telescope. The event rate corresponds to reconstructed showers passing the quality criteria, $\theta$ and $\phi$ is the zenith and azimuth angle of the shower direction respectively and $\omega$ the angle between the reconstructed and the true direction of the shower.

$\mathrm{A} \cap \mathrm{B}$ refers to the combined performance of the HDM of station A and B.

\begin{tabular}{|c|c|c|c|c|c|c|c|}
\hline station & $\begin{array}{l}\text { HDM Position } \\
(\mathrm{x}, \mathrm{y}, \mathrm{z})(\mathrm{m})\end{array}$ & $\begin{array}{l}\text { RFA Position } \\
(\mathrm{x}, \mathrm{y}, \mathrm{z})(\mathrm{m})\end{array}$ & $\begin{array}{l}\text { Event } \\
\text { Rate }\left(\mathrm{hr}^{-1}\right)\end{array}$ & $\begin{array}{l}\sigma_{\theta} \\
(\mathrm{deg})\end{array}$ & $\begin{array}{l}\sigma_{\phi} \\
(\operatorname{deg})\end{array}$ & $\begin{array}{l}\omega_{\text {median }} \\
(\mathrm{deg})\end{array}$ & $\begin{array}{l}E_{t h} \\
(\mathrm{TeV})\end{array}$ \\
\hline A & $\begin{array}{l}(41.2,-64.4,0.5) \\
(35.4,-84.7,0.3) \\
(12.6,-66.2,0.0)\end{array}$ & $\begin{array}{l}(30.5,-70.6,0.4) \\
(82.1,-58.3,1.1) \\
(5.1,-70.2,-0.5) \\
(66.8,-93.7,1.0)\end{array}$ & 17.5 & 3.3 & 10.4 & 3.3 & 20 \\
\hline B & $\begin{array}{l}(219.1,-66.9,9.1) \\
(178.7,-66.3,8.8) \\
(198.7,-73.4,8.5)\end{array}$ & $(193.0,-67.9,9.0)$ & 11.5 & 6.0 & 14.8 & 5.5 & 30 \\
\hline $\mathrm{C}$ & $\begin{array}{l}(-223.8,146.1,4.6) \\
(-211.1,129.0,4.6) \\
(-240.2,130.8,4.6)\end{array}$ & $(-223.6,138.7,4.6)$ & 18.9 & 3.7 & 11.2 & 3.6 & 20 \\
\hline$A \cap B$ & & & 0.15 & 3.6 & 9.5 & 2.9 & $5 \cdot 10^{3}$ \\
\hline
\end{tabular}

The following list of activities have been prepared concerning the HOU cosmic ray telescope:

1. Visits of high school students and teachers to the HOU telescope (University campus in Patras)

2. Lectures for the cosmic rays, the EAS detection techniques and the operation of the telescope

3. "Hands on" experimentation in:

(a) HDM preparation

(b) PMT calibration

(c) Scintillator counter calibration (uniformity tests, MIP signal measurement, timing studies, coincidence studies)

(d) DAQ performance and signal processing

(e) Operation and monitoring of a station

These "hands on" activities are accompanied with presentations covering the theoretical background, the experimental setup, the step by step procedure to do the experiment and the use of the software developed for each activity. Although these educational activities are supposed to take place at the Physics Laboratory of HOU, some of them can be executed remotely, involving thus students from all over the country.

The first activity following the introductory lectures for the telescope is the construction of an HDM. For this purpose, a wooden frame is already prepared that will host the scintillation tiles. Students prepare the reflective paper that surrounds the scintillator and the scintillator tiles and they position them inside the wooden frame. They also prepare and insert the WLS fibers and also attach the WLS common cylindrical junction to the PMT window (figure 3).

One of the activities during the preparation of the array is the PMT calibration. For this activity we use a pulser to drive an LED that illuminates the PMT inside a lightproof box (figure 4). The PMT pulses are acquired by a high sampling rate oscilloscope triggered by the pulser as well. The power supply and the oscilloscope are remotely computer operated from a nearby room in order that the 


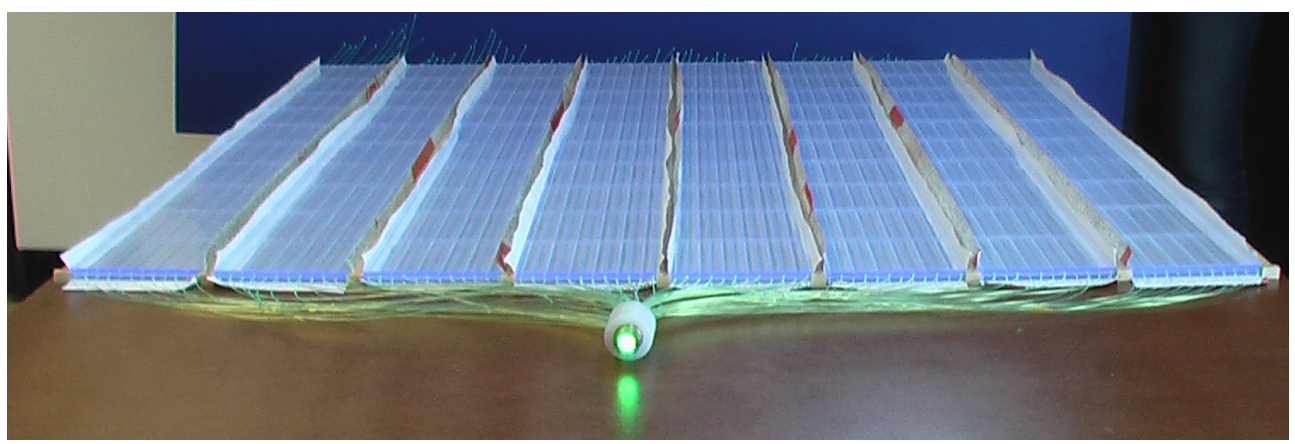

Figure 3. HDM preparation: The 160 scintillation tiles positioned in the wooden frame and the WLS fibres connected to the cylindrical junction.

students are protected from high voltage connectors. In addition, students do not have direct access to expensive equipment preventing thus any accidental damage. By changing the PMT High Voltage with the relevant software students take measurements for the estimation of the slope of the gain vs voltage in logarithmic scale. By increasing the distance between the PMT and the LED and/or using special filters the light intensity on the PMT is reduced, reaching the single photoelectron conditions. The single pe characteristics are measured at a high voltage of $1500 \mathrm{~V}$ and can be extrapolated to any operating voltage. The activity is also designed to be used as a Tele-laboratory by moving with a motor the distance between the LED and the PMT.
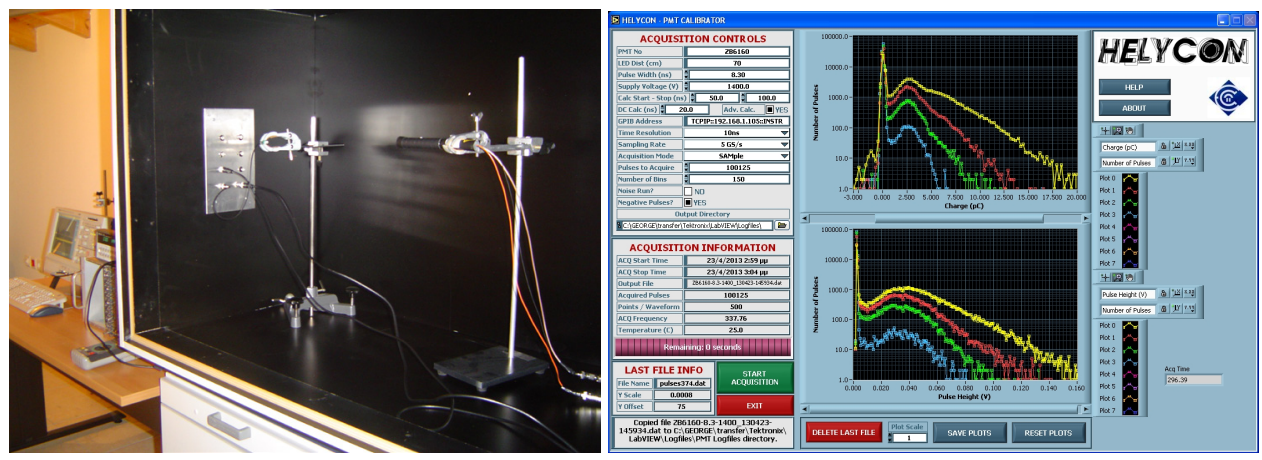

Figure 4. HOU PMT Calibration Setup: On the left photo the lightproof box with the LED (left side) that illuminates the PMT (right side) is shown. A screenshot on the right shows the developed software to operate remotely the oscilloscope and acquire data.

The next activity is the study of the scintillator counter. A simple hodoscope ${ }^{4}$ is used to select MIPs (muons) and it is placed above the counters in nine certain positions. The hodoscope provides the trigger to a high sampling rate oscilloscope and the acquired pulses are analysed. Taking as a reference one of the nine positions the relative strength of the signal as a function of the position is estimated (figure 5). In addition, the relative timing between the signals of the counters as well as

\footnotetext{
${ }^{4}$ Two small scintillator detectors separated by $10 \mathrm{~cm}$ of lead.
} 
the delays of the signal as a function of the position of the incident muon on a single counter can be estimated. As in the case of the PMT calibration, the whole procedure (except the positioning of the hodoscope which takes place with no high voltage supply) is computer operated from another room.
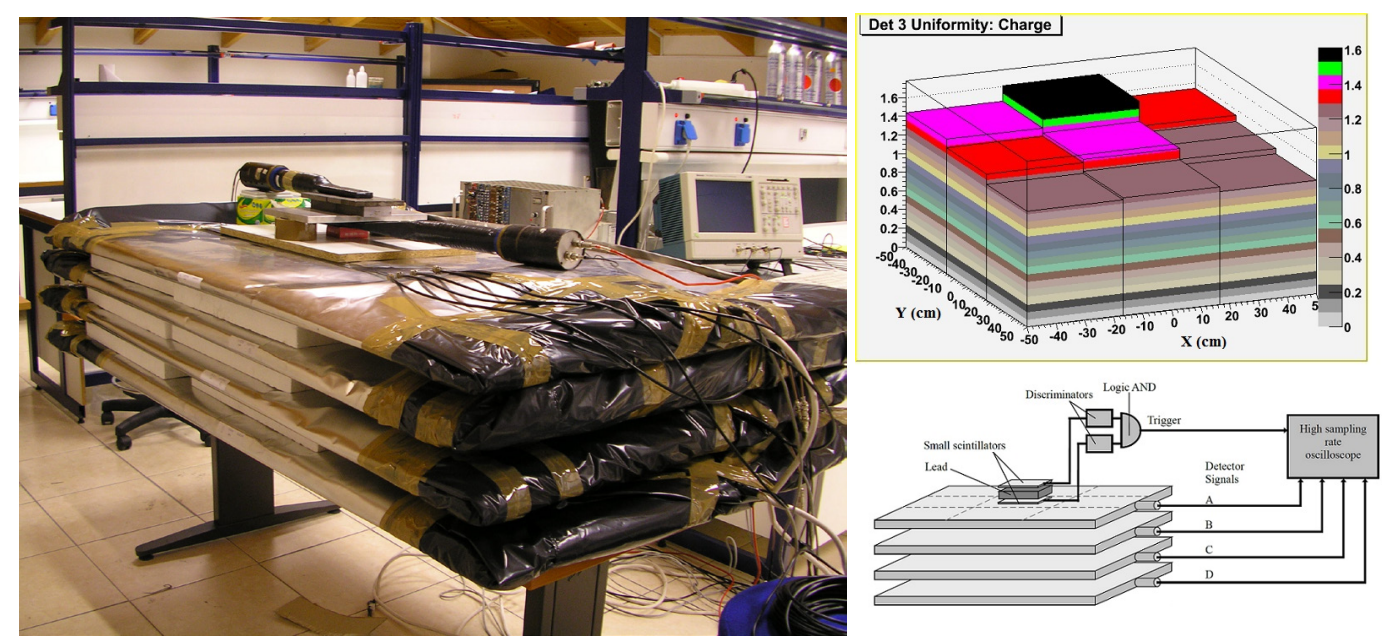

Figure 5. HOU Scintillation Counter Calibration Setup: On the left photo the hodoscope above four HDM is shown. On the right top plot the relative strength from single muons as a function of the position in the local reference system, where the origin is the geometrical centre of the scintillation material, is shown. On the right bottom sketch the experimental setup of the activity is depicted.

The calibration of the DAQ system is an activity that does not require the presence of the students at the Laboratory. The HOU Physics Laboratory has installed three HDM inside a big room, where the waveforms of the PMTs are split and acquired by two separate DAQ systems (figure 6). The first uses a high sampling rate oscilloscope [16], while the other uses a Quarknet board. Both systems are operated remotely using the relevant software. By comparing event by event the information of the two systems a lot of tests can be performed [9]. These include the determination of the exact value of the applied threshold and the timing and ToT resolution. The slewing effect and the corresponding correction as well as the effect of the threshold values to the trigger rate are also part of this activity. Finally, the estimation of the pulse height and of the collected charge as a function of the ToT value at different threshold values can be used to introduce the students to signal processing.

The operation of the station along with the online monitoring is another activity that can be performed remotely as well. During this activity the students operate a station according to the results of the previous results. They apply the nominal high voltage to the HDM, they adjust the threshold values of the DAQ system and start to acquire data. Trigger rates, angular distributions, timing distributions, ToT distributions are compared to reference histograms. Application of different operational parameters like high voltage, threshold values or the level of coincidence and the prediction of the effects are also part of this activity. Furthermore, the shower reconstruction procedure using triangulation and the online event display animation is another activity that can be performed by the students (figure 7).

Finally, additional offline activities are foreseen using data or Monte Carlo events. The accumulated data from the stations can be used by the students for studies like day-night correlations, atmospheric pressure - trigger rate correlations, east-west anisotropy and zenith angle dependence of 


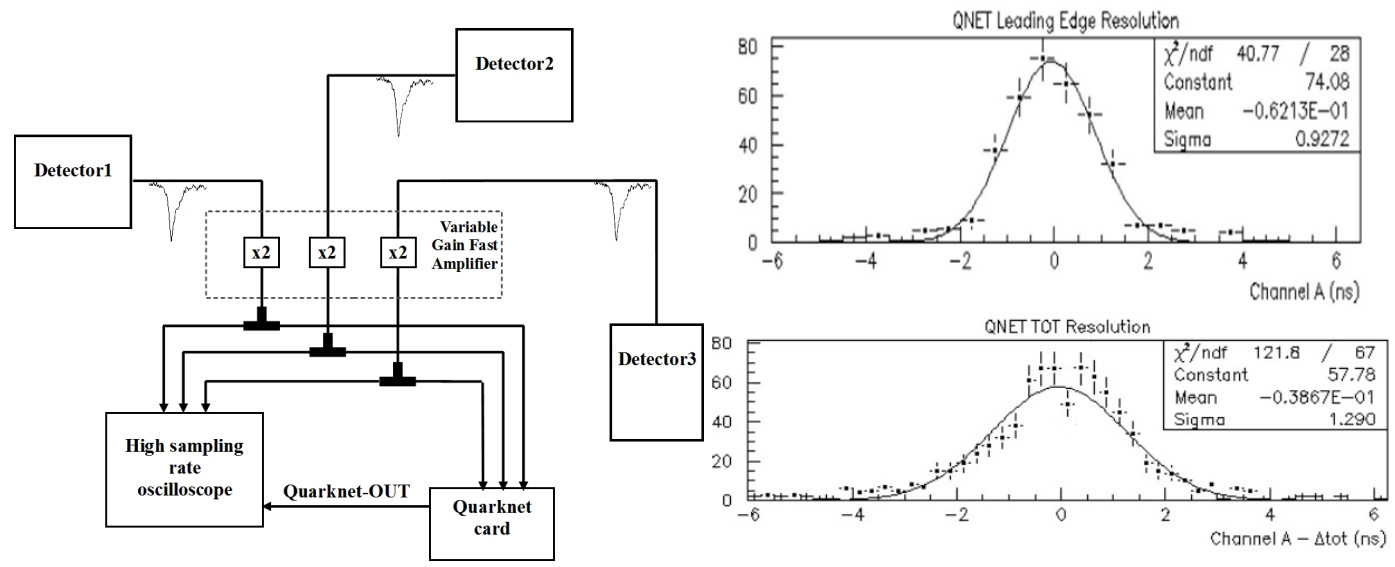

Figure 6. HOU DAQ calibration: On the left the schematic sketch of the setup is shown. The waveforms of the HDM are captured by both the Quarknet board and a high sampling rate oscilloscope. By comparing the the full waveform and the Quarknet data the resolution of the threshold crossing time and the resolution of the ToT value can be estimated.
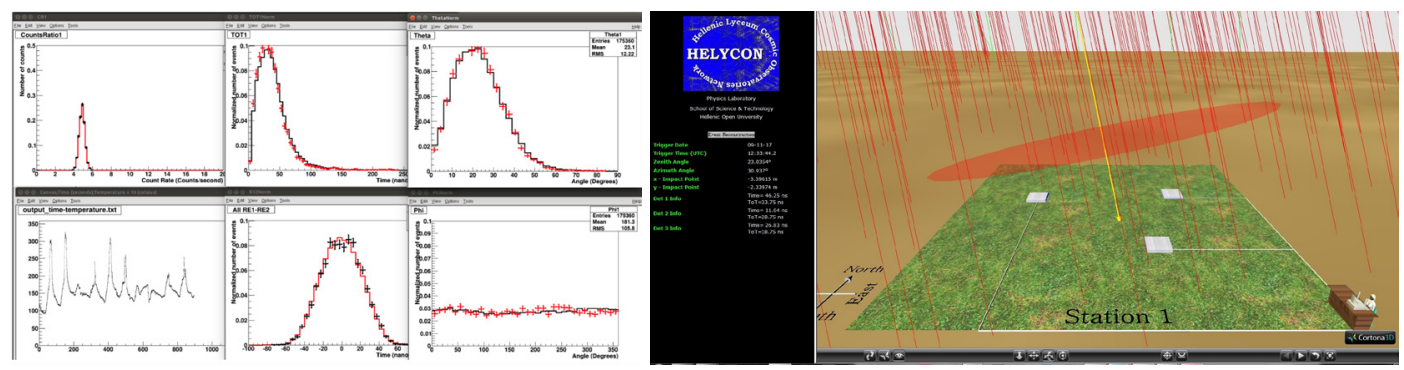

Figure 7. HOU Station Online Monitoring: The Online Monitoring tools include reference histograms to monitor trigger rates, angular and timing distributions as well as environmental data (left plots). On the right the online 3D presenter of the reconstructed showers along with the information provided by the Quarknet board is presented.

the flux. Full Monte Carlo simulations can be used to predict the trigger rates, the efficiency and the angular resolution of different station geometries. Toy Monte Carlo applications using spreadsheets can be used within the classroom or in the computer room of high schools to reproduce the angular distributions and the resolution of the station geometries used in the HOU telescope. More detectors and different spacing configurations can be investigated quickly and easily by the students.

The HOU Physics Lab is collaborating with the Western Greece department of the Ministry of Education and high schools teachers. A student training program is scheduled for the Winter-Spring of 2018. The physics teachers from high schools will visit the HOU Physics Laboratory to get familiar with the experimental procedures and the educational activities described. There will also be seminars at the high schools and 20 students (16-18 years old) will be arranged in four groups and will be trained for a week at the Physics Laboratory of HOU. The students are expected to spend four hours per weekday at the lab. The four groups will be responsible for HDM preparation (1st group), PMT calibration (2nd group), scintillator counter calibration (3rd group) and DAQ calibration (4th 
group). All groups will operate and monitor a station and they will analyse the collected data (shower reconstruction). After the training program the students will prepare presentations of their work and a workshop will be organised where they will present their results to other high school students and to the public.

\section{Summary}

We have deployed three HELYCON stations (each one including three scintillator detectors and one or more CODALEMA antennas) at the Hellenic Open University campus. Each station is autonomous, bearing its own control and monitor electronics and DAQ system and operates continuously. A wide outreach program has started utilising the established procedures of construction, calibration and operation of the stations. Software packages and educational material have been developed and many educational activities have been designed for high school teachers and students.

\section{Acknowledgements}

The preparation, installation and operation of the HOU Telescope has been co-financed by the European Union (European Social Fund - ESF) and Greek national funds through the Operational Program "Education and Lifelong Learning” of the National Strategic Reference Framework (NSRF) - Research Funding Program: “THALIS - Hellenic Open University - Development and Applications of Novel Instrumentation and Experimental Methods in Astroparticle Physics".

The maintenance and the continuation of the operation of the Telescope as well as the outreach program is supported by the Hellenic Open University Grant No. ФK 228.

We would like to thank Michael Petropoulos, George Georgis and the technical staff of HOU for their precious support in this work.

\section{References}

[1] See for example:

VICTA (https://www.uvic.ca/science/physics/vispa/outreach/victa/index.php),

TRIUMF (http://www.triumf.ca/headlines/current-events/triumf-science-education-outreach), QuarkNet (https://quarknet.i2u2.org/home),

WALTA (http://neutrino.phys.washington.edu/ walta/),

T.W.Lynn et al, (CHICOS) arXiv:astro-ph/0509256,

CROP (http://crop.unl.edu),

SALTA (https://faculty.washington.edu/wilkes/salta/),

CLASA (http://physicsweb.phy.uic.edu/quarknet/),

L.A. Anchordoqui, et al, (SCROD) arXiv:hep-ex/0106002,

The Cosmic Ray Project

(https://www.kcl.ac.uk/nms/depts/physics/newsold/events/TheCosmicRayProject.aspx),

QuarkNet Cymru (http://blogs.cardiff.ac.uk/physicsoutreach/2016/11/03/quarknet-cymru/),

Sky-View (http://skyview.uni-wuppertal.de/index.html),

Cosmic@Web

(https://physik-begreifen-zeuthen.desy.de/angebote/kosmische_teilchen/cosmicweb/index_ger.html),

TheNetzwerkTeilchenwelt (http://www.teilchenwelt.de),

Cosmos a 1 Ecole (http://www.sciencesalecole.org/plan-cosmos-a-lecole-materiel/),

e-PERON (https://www.labex-ocevu.univ-amu.fr/?q=fr/content/e-peron), 
HiSPARC (http://www.hisparc.nl/en/),

SEASA (http://gluon.particle.kth.se/SEASA/ ),

MAZE (http://maze.u.lodz.pl/ang/index.html),

CZELTA (http://czelta.utef.cvut.cz/publicweb/?language=en),

SKALTA (http://astronomy.science.upjs.sk),

HELYCON (http://helycon.eap.gr),

Abbrescia et. al., (EEE) Journal of Physics: Conference Series 718 (2016)

Maria Krause et al, arXiv:1508.03968 [astro-ph.IM]

[2] S.E. Tzamarias, HELYCON: towards a sea-top infrastructure (Proceedings of the 6th International Workshop on the Identification of Dark Matter (IDM 2006), World Scientific (2007), ISBN13978-981-270-852-6)

[3] A. Leisos et al, Nucl. Instr. and Meth. A, 595, 80-83 (2008)

[4] KM3Net Collaboration, KM3NeT-TDR (ISBN 978-90-6488-033-9)

[5] S. Hansen et al, IEEE Transactions on Nuclear Science, 51 Issue: 3 (2004)

[6] Konstantina Georgakopoulou et al, arXiv:1702.01066 [physics.ins-det]

[7] D. Ardouin A. Belletoile D. Charrier et al, Nucl. Instr. and Meth. A, 555, 148-163 (2005)

[8] I. Manthos et al, arXiv:1702.05794 [physics.ins-det]

[9] T. Avgitas et al, arXiv:1702.04902 [physics.ins-det]

[10] G. Bourlis et al, Nucl. Instr. and Meth. A, 626-627, 163-165 (2011)

[11] A.G. Tsirigotis et al, Nucl. Instr. and Meth. A, 626, 185-187 (2011)

[12] A.G. Tsirigotis et al, Nucl. Instr. and Meth. A, 725, 68-71 (2013)

[13] D. Heck et al, Forschungszentrum Karlsruhe Report, (1998)

[14] S.Agostinelli et al, Nuclear Instruments and Methods in Physics Research A 506 (2003)

[15] T. Avgitas et al, Operation of a pilot HELYCON cosmic ray telescope with 3 stations, to be published

[16] G. Bourlis, et al, A Data Acquisition System based on high sampling rate oscilloscopes PCI '16 Proceedings of the 20th Pan-Hellenic Conference on Informatics, ISBN: 978-1-4503-4789-1 2016. 